\title{
HVDC Control Strategies to Improve Transient Stability in Interconnected Power Systems
}

\author{
J. Hazra \\ Dpt of Power and Energy Systems, \\ SUPELEC, France, \\ jagabondhu.hazra@supelec.fr
}

\author{
Y. Phulpin \\ Dpt of Power and Energy Systems, \\ SUPELEC, France, \\ yannick.phulpin@supelec.fr
}

\author{
D. Ernst \\ Research Associate, FNRS \\ University of Liège, Belgium, \\ dernst@ulg.ac.be
}

\begin{abstract}
This paper presents three HVDC modulation strategies to improve transient stability in an interconnected power system. AC variables such as rotor speeds, voltage phasors, and tieline power flows are used as input to the controller that modifies the power flow settings through the HVDC-links. The proposed techniques are tested on the IEEE 24-Bus reliability test system and critical clearing times obtained for several contingencies are analyzed. The paper shows that HVDC modulation can lead to substantial improvement in transient stability.
\end{abstract}

\section{INTRODUCTION}

In the aftermath of severe power system disturbances, transient stability issues such as loss of synchronism phenomena may occur [1]. Those phenomena may have for effect to lead in a very short period of time to the loss of many synchronous generators and, in turn, endanger the stability of the whole system [2]. Numerous control schemes have thus been proposed to mitigate loss of synchronism phenomena. They can be broadly classified into two groups, i.e. preventive control and emergency control. Whereas preventive control anticipates events to ensure that the power system is to withstand the most critical events, emergency control is executed immediately after the disturbances.

Much research has been carried out to design effective emergency control schemes. By way of example, an emergency generator and load shedding scheme based on the topological and dynamic characterization of stability boundary is proposed in [3]. Reference [4] proposes an on-line emergency control strategy for generator shedding based on PMU measurements. An open-loop emergency control scheme based on generation rescheduling and/or generation tripping is developed in [5]. Real-time closed-loop emergency control schemes for generation shedding are also presented in [6], [7]. These methods compute corrective actions by using equal area type criteria together with real-time measurements.

Usually, emergency control techniques involve actions on generators and/or loads to maintain stability. In addition, flexible transmission devices such as fast valving and breaking resistors [8], fast excitation controllers [9], or tie-line reactance controllers [10] could also be used as means for emergency control of transient stability. In power systems where HVDClinks have been installed, a better alternative could be to use those devices as emergency control means. As AC/DC conversion involves no inertia and power settings of HVDClinks can be changed quasi-instantaneously [11], modulating of active power flow through the HVDC-links has been proposed to improve power system stability. By way of example, References [12]-[15] present modulation techniques for stability purpose.

When an HVDC-link replaces a conventional AC line, this alternative is even necessary, as DC transmission devices do not offer the damping abilities of $\mathrm{AC}$ transmission lines when they are operated in a constant power mode. In this paper, we propose to study how the power flow settings of the HVDClinks can be controlled to increase transient stability margins. Four control strategies are considered and their effectiveness is analyzed. First, the HVDC control settings are unchanged during the disturbance. Second, the power flow through the HVDC-link is modulated based on the rotor speed at the two sides of the HVDC-link. Third, the modulation is based on the phase angle difference between the inverter and converter side buses. Fourth, the modulation is based on the rate of change of the power flow between the control areas where the HVDC end buses are located. The performance obtained with the different control strategies is evaluated on the IEEE 24 bus system with two HVDC links.

The paper is organized as follows. Section II details the proposed control strategies, Section III presents the benchmark power system, Section IV presents the simulation results, and Section V concludes.

\section{CONTROL SCHEME FOR HVDC-LINK MODULATION}

This paper focuses on four control strategies for the HVDClink, where the inputs are based on different AC variables. It is supposed that those variables are known instantaneously and no information delay is considered. The control variables are the power flow settings $P_{D C}$ for each HVDC-link. The settings are subjected to limitations with respect to a maximum and minimum value of the power flow. In addition, as introduced in [16] to reduce the risk of commutation failure and voltage instability, $P_{D C}$ is automatically reduced when the DC voltage at the rectifier bus is below a specified threshold value. 
For each controllable HVDC-link, the power flow setting is derived from a specific input signal $e$ by a proportional integral controller, which can be mathematically written as follows

$$
\begin{array}{r}
P_{D C_{s e t}}=K_{p} \cdot e+K_{i} \int e \cdot d t \\
P_{D C}=P_{D C_{0}}+P_{D C_{s e t}},
\end{array}
$$

where $K_{p}$ and $K_{i}$ are the proportional and integral coefficients and $P_{D C_{0}}$ is the reference value for the HVDC power flow, which is to be determined by a secondary control level with a larger time constant. We refer to References [17], [18] for some practical examples of such secondary control levels.

The four control strategies to compute the HVDC settings $P_{D C_{s e t}}$ based on different input signals $e$ are described hereafter.

\section{A. Strategy 1}

With strategy $1, e$ is equal to zero, i.e. no specific power modulation control is applied to the HVDC system. Nevertheless, $P_{D C}$ is subjected to the limitations introduced here above.

\section{B. Strategy 2}

With strategy 2, $P_{D C_{s e t}}$ is modulated based on the rotor speed of the generators located closest to the rectifier and inverter buses of the HVDC-link, respectively.

As emphasized in [19], this type of input signal may have for effect to transfer more power from the area where the machines are accelerating faster, which should mitigate this acceleration and therefore improve transient stability in the system. The error signal $e$ is derived as follows

$$
e=\frac{d}{d t}\left(w_{D C_{r}}-w_{D C_{i}}\right)
$$

where $w_{D C_{r}}$ and $w_{D C_{i}}$ are the rotor speeds of the rectifier and inverter buses of the HVDC-link, respectively.

\section{Strategy 3}

With strategy $3, P_{D C_{\text {set }}}$ is modulated based on the voltage phase angle at the end buses of the HVDC-link. The rationale of this control strategy is that the difference of voltage phase angle at the end buses of the HVDC-link may give a good image of the mechanical angles of the generators located closest to those buses. The voltage phase angles can be measured through phasor measurement units, for example.

The error signal $e$ is computed as follows

$$
e=\frac{d}{d t}\left(\delta_{D C_{r}}-\delta_{D C_{i}}\right)
$$

where $\delta_{D C_{r}}$ and $\delta_{D C_{i}}$ are the voltage phase angle at the rectifier and inverter side buses of the HVDC-link, respectively.

\section{Strategy 4}

With strategy $4, P_{D C_{\text {set }}}$ is modulated based on the AC power flow in the inter-area tie lines. This control scheme supposes the definition of well-defined control areas interconnected by the HVDC-links. The input signal $e$ is based on the sum of the active power flows between the control areas where the converter and inverter buses are located, which is represented by $P_{I A}$.

$$
e=\frac{d}{d t}\left(P_{I A}\right)
$$

\section{DESCRIPTION OF THE BENCHMARK POWER SYSTEM}

This section first introduces the models of the different power system elements of the IEEE 24 bus system that will be used later in the simulations. Second, the numerical program chosen to simulate the system is described.

\section{A. AC system representation}

1) Generator model: In the benchmark system, the generators are synchronous machines with constant mechanical power. The electrical part of the generator is represented by a constant voltage source behind a transient reactance. This model has often been chosen in transient stability studies [20], [21].

2) Load model: Load demands are considered voltage dependent in the benchmark system. In particular, the real and reactive power demand at bus $i\left(P_{i}\right.$ and $Q_{i}$, respectively) are expressed as follows

$$
\begin{aligned}
& P_{i}=P_{i_{0}}\left(a_{i}+b_{i} \cdot V_{i}+c_{i} \cdot V_{i}^{2}\right), \\
& Q_{i}=Q_{i_{0}}\left(a_{i}^{\prime}+b_{i}^{\prime} \cdot V_{i}+c_{i}^{\prime} \cdot V_{i}^{2}\right),
\end{aligned}
$$

where $P_{i_{0}}$ and $Q_{i_{0}}$ are the active and reactive power demand at bus $i$ at nominal voltage, respectively, $a_{i}, b_{i}$, and $c_{i}$ are the coefficients of active power demand at bus $i$ and $a_{i}^{\prime}, b_{i}^{\prime}$, and $c_{i}^{\prime}$ are the coefficients of reactive power demand at bus $i$.

3) Transmission line model: The transmission lines are represented by constant admittances.

\section{B. HVDC-link representation}

The HVDC power electronics dynamics is neglected in this study, as its time constant is much smaller than those involved in transient stability phenomena [22]. The power flow $P_{D C}$ through each HVDC-link is represented as a positive load at the rectifier bus, and negative load at the inverter bus. The parameters of those elements are function of the rectifier and inverter side $\mathrm{AC}$ bus voltages, rectifier and inverter side transformer tap positions, and internal HVDC parameters, i.e. converter firing angle $\alpha$, inverter extinction angle $\gamma$, and current in the DC line.

Three modes of operation are designed to compute the power injections from the end buses of each HVDC-link [2]. Under normal conditions, the HVDC-links operate in constant power mode when the rectifier operates in constant current control and the inverter operates in constant extinction angle control. During disturbances, when $\alpha$ reaches extreme values, 
the mode of operation changes. The control modes can be written as follows.

- Mode 1: Constant power/current control at the rectifier and constant extinction angle at the inverter.

- Mode 2: Minimum firing angle control at the rectifier and constant power/current control at the inverter.

- Mode 3: Minimum firing angle control at the rectifier, and constant extinction control at the inverter. This is the transition mode between Mode 1 and Mode 2.

\section{Computation tool for time-domain analysis}

Most transient stability analysis methods represent HVDClinks as constant power loads regardless of their response during disturbances. However, in this study, HVDC-links are treated as non-linear variable transmission devices. To incorporate their features, a specific algorithm has been developed, where load flows are sequentially solved at each simulation step to update the injections at the rectifier and inverter buses.

As emphasized in [23], Newton-Raphson method may be used to solve the network equations. However, this method is computationally expensive because it needs to calculate the Jacobian at each iteration of the algorithm. To improve the computational performance, a fast decoupled solution technique is used to solve AC and DC network equations iteratively. The time-domain computation technique developed for transient stability evaluation of AC/DC system is as follows.

1) Solve the load flow problem to obtain bus voltages.

2) Solve Mode 1 DC equations.

(a) If $\alpha>\alpha_{\min }$, go to step 3 .

(b) If $\alpha \leq \alpha_{\min }$, solve Mode 2 equations. If $\gamma>\gamma_{\min }$, go to step 3 .

(c) If $\gamma \leq \gamma_{\text {min }}$, solve Mode 3 .

3) Compute the real and reactive power injections at each DC terminal. If the mismatch is greater than the predefined tolerance, go back to step 1 and solve $\mathrm{AC}$ equations.

4) Using the bus voltages, calculate generator currents and electrical outputs.

5) Solve generator swing equations using trapezoidal rule [24] and update the voltages behind the transient reactance by changing their phase angles.

6) Implement changes in the HVDC settings based on the control scheme under investigation.

7) If the maximum simulation time has not been reached, repeat the simulation procedure from step 1 .

To improve computational time, the sparsity technique introduced in [25] has been used to solve the network equations.

\section{Simulation Results}

Simulations have been carried out on the IEEE 24 bus reliability test system with two bipolar HVDC-links added as shown in Fig. 1. One link is located between bus 21 and bus 11 , and the other one is between bus 18 and bus 3 . The main pre-fault load-flow information are provided in Tables I-III.

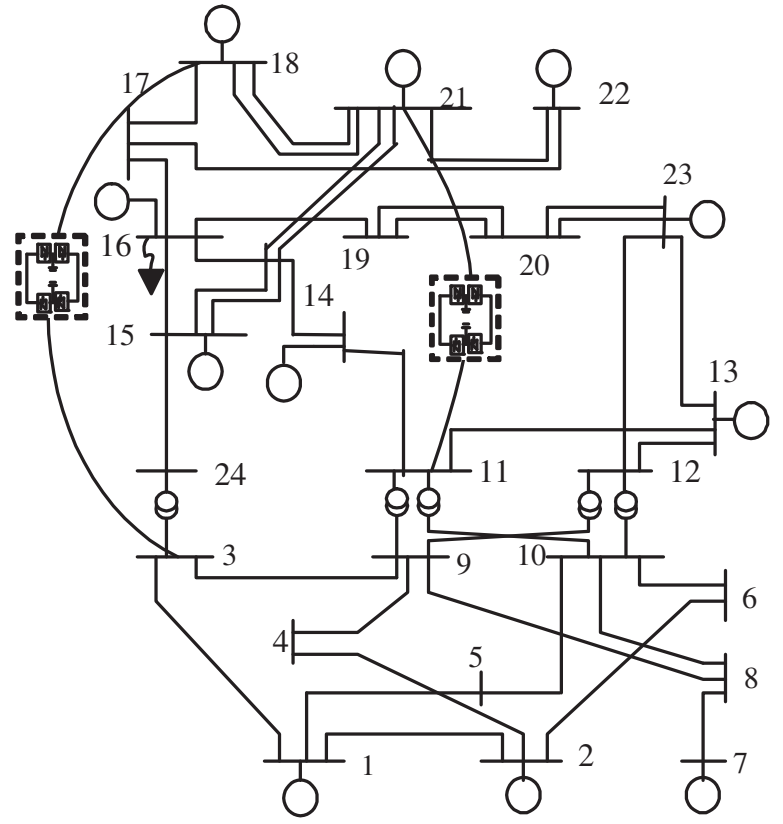

Fig. 1. IEEE 24 bus RTS-96 system with two HVDC-links.

TABLE I

PRE-CONTINGENCY GENERATOR OUTPUTS.

\begin{tabular}{|c|c|c|c|}
\hline Bus number & $\begin{array}{c}P_{i} \\
\text { (MW) }\end{array}$ & $\begin{array}{c}Q_{i} \\
\text { (MVAR) }\end{array}$ & $\begin{array}{c}V_{i} \\
\text { (p.u.) }\end{array}$ \\
\hline 1 & 161 & 31 & 1.025 \\
\hline 2 & 161 & 24 & 1.025 \\
\hline 7 & 257 & 61 & 1.025 \\
\hline 13 & 525 & 102 & 1.025 \\
\hline 15 & 187 & -2 & 1.000 \\
\hline 16 & 133 & -50 & 1.000 \\
\hline 18 & 363 & 145 & 1.025 \\
\hline 21 & 363 & 122 & 1.025 \\
\hline 22 & 280 & 31 & 1.025 \\
\hline 23 & 446 & 87 & 1.035 \\
\hline
\end{tabular}

TABLE II

PRE-CONTINGENCY POWER FLOWS IN THE POWER SYSTEM.

\begin{tabular}{|c|c|c|c|}
\hline From bus & To bus & $P_{\text {from }}(\mathrm{MW})$ & $Q_{\text {from }}(\mathrm{MVAR})$ \\
\hline 16 & 17 & -144 & -49 \\
\hline 15 & 21 & -111 & -40 \\
\hline 15 & 24 & 98 & -2 \\
\hline 11 & 14 & 44 & 10 \\
\hline 23 & 12 & 114 & 16 \\
\hline 23 & 13 & 40 & 16 \\
\hline
\end{tabular}

TABLE III

PRE-CONTINGENCY POWER FLOWS THROUGH THE HVDC-LINKS.

\begin{tabular}{|c|c|c|c|}
\hline Label & From bus & To bus & P from $_{\text {fMW }}$ \\
\hline HDVC 1 & 21 & 11 & 180 \\
\hline HDVC 2 & 18 & 3 & 90 \\
\hline
\end{tabular}




\section{A. Illustrative example}

A three phase to ground fault is applied to bus 16 at $\mathrm{t}=100$ $\mathrm{ms}$, and the fault is cleared by tripping lines 16-14, 16-17 and $16-19$ at $\mathrm{t}=200 \mathrm{~ms}$. As the time frame of interest in transient stability analysis is usually three to five seconds following the disturbance [2], this study is limited to the first five seconds after the short-circuit inception. If the maximum angular difference between two machines goes beyond 180 degrees, the system is assumed unstable.

1) Strategy 1: Simulation results in this section are obtained with no specific control action. System responses due to the bus fault are displayed in Fig. 2-3, which show the power flow through HVDC-links and electromechanical swings of the generators, respectively.

It can be noticed by observing the swing curves that the generators separate into two groups in the aftermath of the disturbance. In particular, generators at buses 18,21 , and 22 get out of step together. Moreover, though $P_{D C_{s e t}}$ remains unchanged for each HVDC-link, $P_{D C}$ oscillates due to the voltage swings, which induce limitations on $P_{D C}$ to avoid commutation failures.

2) Strategy 2: Simulation results obtained with strategy 2 are shown in Fig. 4-5. As introduced in Section II-B, since no generator is located at the southern end bus of the HVDC, the rotor speed of the generator at bus 13 has been associated to the end buses.

With this modulation scheme, the system damps the severe electromechanical swing among the generators and prevents the generators at buses 18, 21, and 22 from going out of step. Therefore, in this case, the system is stable. The performance of the controller is based on the proper tuning of the controller parameters. In this case, those are chosen as follows: $K_{p}=$ 0.01 and $K_{i}=0.001$.

3) Strategy 3: Fig. 6 shows the modulated HVDC signal and Fig. 7 presents the corresponding swing plot of all the generators in the system.

Those figures show that the system is stable when the HVDC modulation is done according to strategy 3 with $K_{p}=0.1$ and $K_{i}=0.001$.

4) Strategy 4: Fig. 8 shows $P_{D C}$ and Fig. 9 shows the generator angles. In this study, $P_{I A}$ is computed as the sum of the active power flows from bus 15 to bus 24,14 to 11,23 to 12 , and 23 to 13 .

Simulation results show that using strategy 4 is effective. In this case, $K_{p}=0.02$ and $K_{i}=0.001$.

\section{B. Effectiveness of the control strategies}

The effectiveness of the proposed control strategies is analyzed in terms of critical clearing time (CCT). This index represents the maximum short-circuit duration the power system can sustain without loosing synchronism [2]. As most of the methods for computing CCT do not consider control actions, the CCTs are computed by using a dichotomy search.

The effects of four different contingencies have been analyzed, and simulation results are reported in Table IV. It can be observed that the lowest CCT is obtained with strategy 1 for
TABLE IV

CRITICAL CLEARING TIME (IN MS) FOR DIFFERENT CONTINGENCIES WITH THE PROPOSED STRATEGIES.

\begin{tabular}{|c|c|c|c|c|}
\hline Contingency & Strategy 1 & Strategy 2 & Strategy 3 & Strategy 4 \\
\hline Bus 16, Line 16-14 & 183 & 288 & 307 & 321 \\
\hline Bus 18, Line 18-21 & 195 & 325 & 336 & 365 \\
\hline Bus 15, Line 15-21 & 280 & 291 & 305 & 316 \\
\hline Bus 6, Line 6-2 & 453 & 469 & 462 & 526 \\
\hline
\end{tabular}

every contingency considered. On the other hand, significantly larger CCTs are obtained with the alternative strategies. In particular, strategy 4 leads to the highest CCT for every contingency under consideration. The improvement is however limited (10 through $70 \mathrm{~ms}$ ) when the short-circuits are located at buses 15 and 6 . In those cases, it might be supposed that the improvement in CCT would also be limited if an AC line were in the place of each HVDC-link.

\section{CONCLuSions}

This paper presents three HVDC modulation techniques to improve transient stability of interconnected power systems with integrated HVDC systems. The proposed techniques assess the power flow settings for the HVDC-links based on different AC variables. Simulation results show that HVDC modulation significantly increases the stability of the system, which has been measured in our experiences in terms of critical clearing times for different contingencies.

This finding suggests that the proposed emergency control schemes could be effective for systems with HVDC links. However, before applying the proposed techniques, important issues need to be addressed. In particular, the assumption of instantaneous knowledge of the $\mathrm{AC}$ variables is certainly optimistic, if not naive, and the impact of communication delays/failures on the emergency control schemes should be investigated. In addition, as the performance obtained with the control strategies depends on the proportional and integral coefficients, a proper selection should be handled to optimally assess their values.

Furthermore, it must be acknowledged that, whereas the test system was tested under different contingencies for which the HVDC-modulation was effective, it is unlikely to obtain the same results for any contingency in the system. It could thus be relevant to design advanced control strategies that could improve transient stability of the system for every possible contingency. Those strategies should certainly rely on coordinated control for power systems with multiple HVDClinks. 


\section{REFERENCES}

[1] M. Pavella, D. Ernst, and D. Ruiz-Vega, Transient Stability of power systems. Kluwer Academic, 2000.

[2] P. Kundur, Power System Stability and Control. McGraw-Hill, 1994.

[3] Y. Min, K. Hou, R. Zhang, and Q. Tu, "A new method for generation shedding and load shedding in power system emergency control," in Proc. IEEE International Conference on Electric Utility Deregulation, Restructuring and Power Technologies, vol. 1, April 2004, pp. 210-214.

[4] C. Li, C. Deng, Y. Sun, and X. Chen, "An on-line transient stability emergency control strategy based on PMU forecasted trajectory," in Proc. International Power Engineering Conference, Dec 2007, pp. 807812.

[5] D. Ruiz-Vega and M. Pavella, "A comprehensive approach to transient stability control. II. open loop emergency control," IEEE Trans Power System, vol. 18, no. 4, pp. 1454-1460, Nov 2003.

[6] D. Ernst and M. Pavella, "Closed-loop transient stability emergency control," in Proc. IEEE Power Engineering Society Winter Meeting, vol. 1, 2000, pp. 58-62.

[7] M. Glavic, D. Ernst, D. Ruiz-Vega, L. Wehenkel, and M. Pavella, "ESIME - a method for transient stability closed-loop emergency control: achievements and prospects," in Proc. iREP Symposium- Bulk Power System Dynamics and Control - VII,Revitalizing Operational Reliability, Aug 2007, pp. 1-10.

[8] R. Patel, T. S. Bhatti, and D. P. Kothari, "A modified approach to transient stability enhancement with fast valving and braking resistor applications," Int J Electrical Power \& Energy Systems, vol. 28, no. 10, pp. 729-738, Dec 2006.

[9] N. Fernandopulle and R. Ramshaw, "Domain of stability of a synchronous machine with excitation control-a cell mapping approach," Electric Power Systems Research, vol. 27, no. 3, pp. 173-181, Aug 1993.

[10] D. K. Reitan and N. Ramarao, "A method of improving transient stability by bang-bang control of tie-line reactance," IEEE Trans Power Apparatus and Systems, vol. PAS-93, no. 1, pp. 303-311, Jan 1974.

[11] N. G. Hingorani and L. Gyugyi, Understanding FACTS: Concepts and Technology of Flexible AC Transmission Systems. Wiley-IEEE Press, 1999.

[12] S. K. Tso and S. P. Cheung, "Fast prediction of transient stability margin in systems with SVC control and HVDC link," in Proc. Int. Conf. Energy Management and Power Delivery, vol. 2, Nov 1995, pp. 456-461.

[13] A. Teshome, "Effect of a modulated HVDC link on power system transients," Electric Power System Research, vol. 25, no. 2, pp. 101109, Nov 1992.

[14] K. Tomiyama, M. Sato, K. Yamaji, M. Sekita, and M. Goto, "Power swing damping control by HVDC power modulation in an ACDC hybrid transmission system," Electrical Engineering in Japan, vol. 124, no. 3, pp. 10-18, Dec 1998.

[15] M. Xiao-ming and Z. Yao, "Application of HVDC modulation in damping electromechanical oscillations," in Proc. IEEE Power Engineering Society General Meeting, June 2007, pp. 1-6.

[16] M. Khatir, H. S. Zidi, Sid Ahmed, and M. K. Fellaf, "Analysis of recovery from commutation failures in an HVDC inverter connected to a weak receiving ac system," Acta Electrotechnica et Informatica, vol. 8, no. 1, pp. 44-50, 2008.

[17] A. Pizano-Martinez, C. R. Fuerte-Esquivel, H. Ambriz-Perez, and E. Acha, "Modeling of VSC-based HVDC systems for a NewtonRaphson OPF algorithm," IEEE Transactions on Power Systems, vol. 22, no. 4, pp. 1794-1803, November 2007.

[18] L. A. S. Pilotto, W. W. Ping, A. R. Carvalho, A. Wey, W. F. Long, F. M. Alvarado, and A. Edris, "Determination of needed FACTS controller that increase asset utilization of power systems," IEEE Transactions on Power Delivery, vol. 12, no. 1, pp. 364-371, January 1997.

[19] R. Eriksson, V. Knazkins, and L. Soder, "On the assessment of the impact of a conventional HVDC on a test power system," in Proc. 2007 iREP Symposium - Bulk Power System Dynamics and ControlVII, Revitalizing Operational Reliability, Aug 2007.

[20] M. A. Pai, Energy Function Analysis for Power System Stability. Kluwer Academic Publishers Group, 1989.

[21] M. Pavella and P. G. Murthy, Transient Stability of Power Systems: Theory and Practice. Wiley, 1993.

[22] X. Y. Ni and A. A. Fouad, "A simplified two-terminal de model and its use in direct transient stability assessment," IEEE Trans Power System, vol. 2, no. 4, pp. 1006-1012, Nov 1987.
[23] Y. A. Mobarak, "Modified load flow analysis for integrated ACDC power systems," in Proc. 12th International Middle-East Power System Conference, Mar 2008, pp. 402-405.

[24] A. Kaw and K. M, An interactive e-book for illustrating the Trapezoidal Rule for numerical integration. Holistic Numerical Methods Institute, University of South Florida, 2004

[25] K. Zollenkopf, Large Sparse Sets of Linear Equations, ch. Bifactorization-Basic computational algorithm and programming techniques. New York: Academic Press, 1971. 


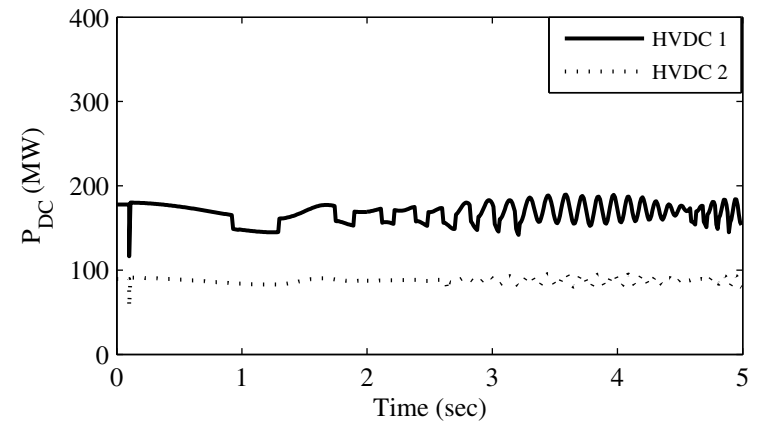

Fig. 2. Power flow through the HVDC-link with strategy 1.

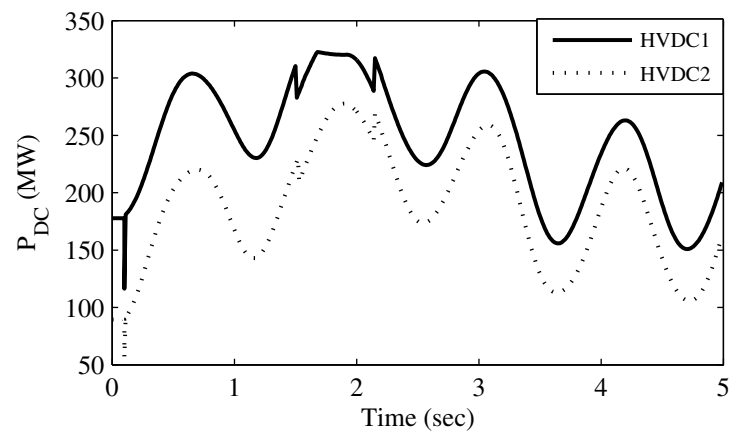

Fig. 4. Power flow through the HVDC-link with strategy 2.

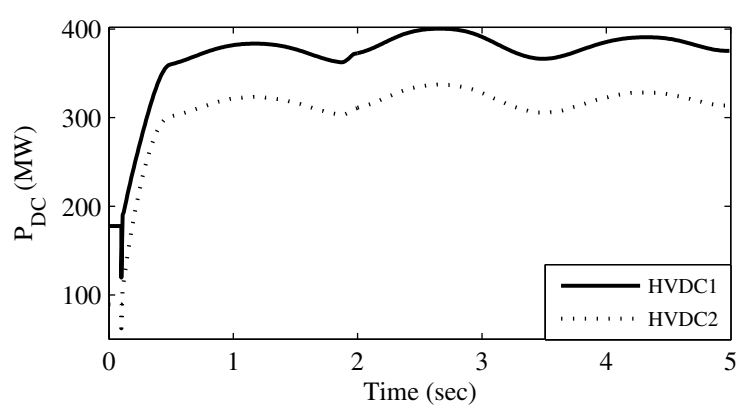

Fig. 6. Power flow through the HVDC-link with strategy 3.

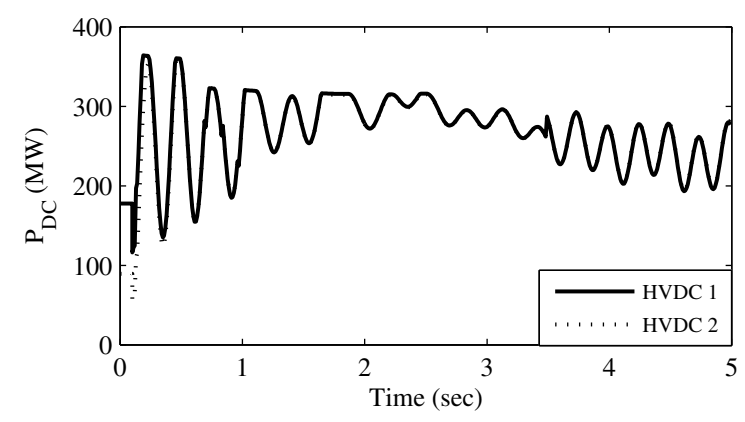

Fig. 8. Power flow through the HVDC-link with strategy 4.

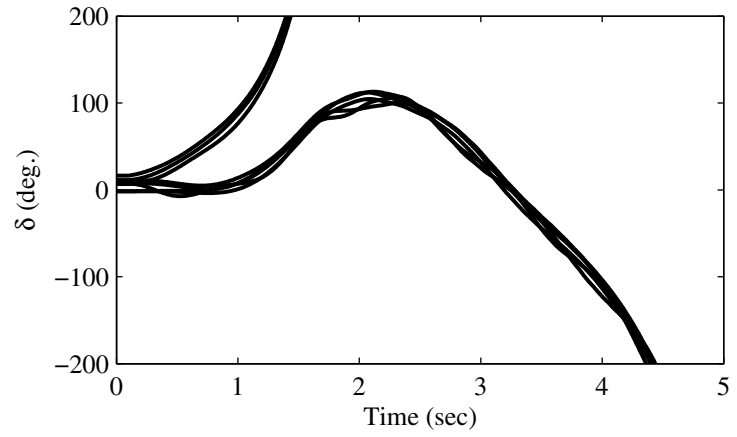

Fig. 3. Generators swing plot with strategy 1.

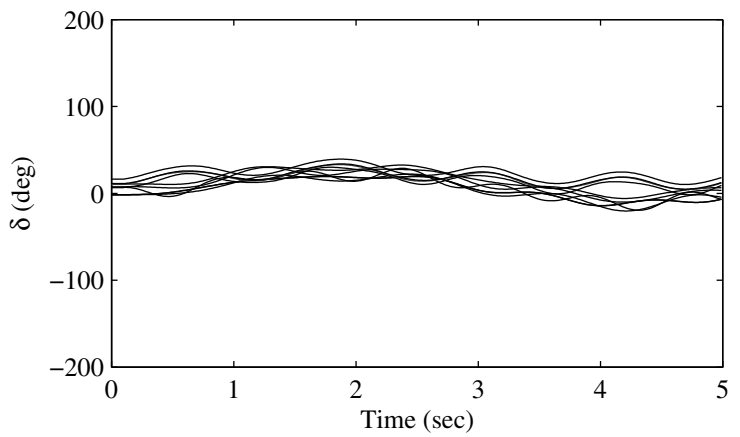

Fig. 5. Generators swing plot with strategy 2.

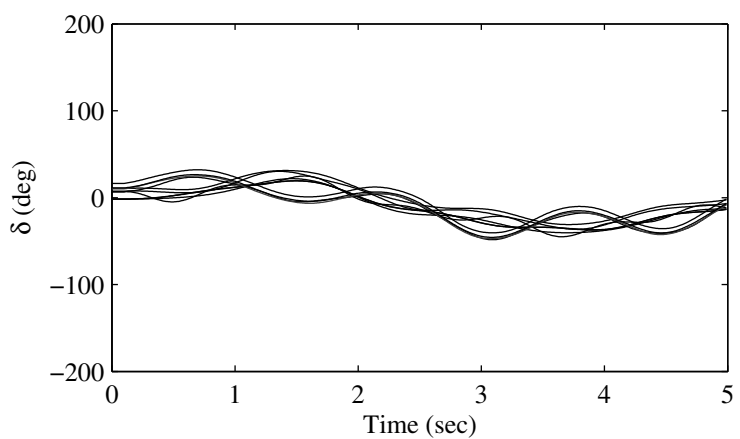

Fig. 7. Generators swing plot with strategy 3.

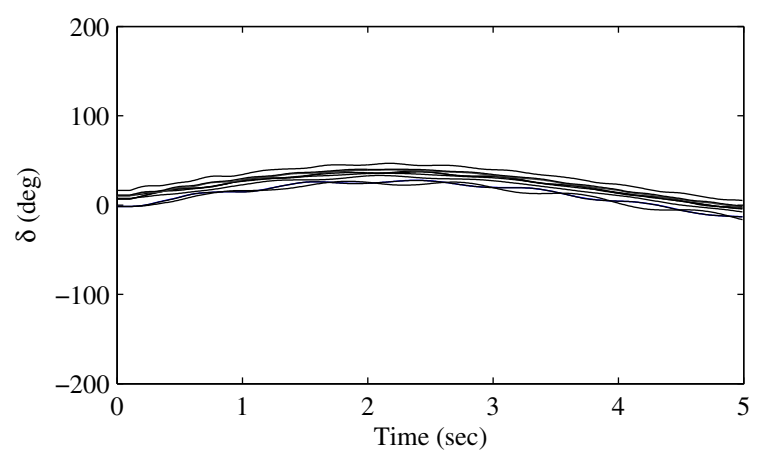

Fig. 9. Generators swing plot with strategy 4. 